\title{
Bilateral macular neurosensory retinal detachment secondary to systemic Dabrafenib and Trametinib therapy
}

\author{
Kevin J. Talbot*, and Gibran Khurshid \\ Department of Ophthalmology, college of medicine, University of Florida, 1600 SW Archer Road, Gainesville, FL 32610, USA
}

\begin{abstract}
We report the case of a patient who developed bilateral macular neurosensory retinal detachment from dabrafenib and trametinib (B-Raf inhibitor and MEK1/2 inhibitor, respectively) for stage IV cutaneous melanoma. To the best of our knowledge, this has not previously been reported as a side-effect of the simultaneous use of these two medication.
\end{abstract}

A 45 year old Caucasian female presented with bilateral macular edema. She had a history of cutaneous melanoma of her right lower extremity that was diagnosed 5 years ago. After excision, sentinel lymph node biopsy was positive and she underwent subsequent lymphadenectomy. She remained in remission for more than 3 years until recurrence, and subsequently was classified as having stage IV melanoma. At that time, she was started on dabrafenib 150mg BID and trametinib $2 \mathrm{mg}$. Her past ocular history was unremarkable. Three weeks prior to our visit, she was seen by another ophthalmologist for her yearly eye exam and although the patient was without vision complaints, macular edema was noticed on macular Ocular Coherence Tomography (Figure 1). A thorough review of systems was negative for any known cause of macular edema, and given the recent addition of these medications, it was thought that these could have been the cause of the macular edema. After discussion with the prescribing provider, these medications were discontinued. Approximately 3 weeks later, the patient still was without any eye complaints, had a visual acuity of 20/25 OD and 20/20-2 OS, and normal complete eye exam. The only change in medication was the aforementioned stoppage of dabrafenib and trametinib. Repeat Macular OCT showed resolution of the previously noted neurosensory detachment and is shown below (Figure 2).

\section{Discussion}

Dabrafenib acts as an inhibitor of the enzyme B-Raf, which plays

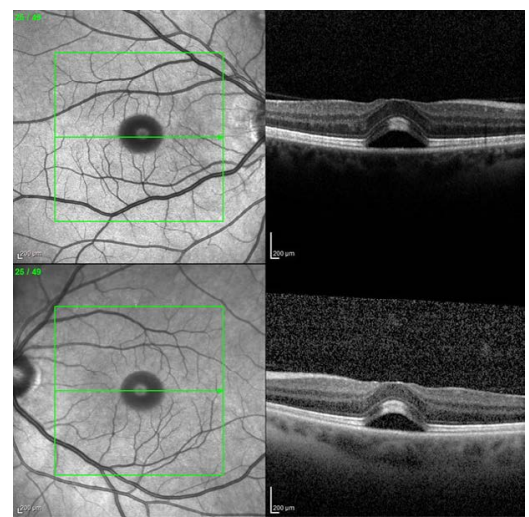

Figure 1. Bilateral sub-foveal neurosensory retinal detachment. a role in the regulation of cellular proliferation and is part of the MAP kinase pathway. Dabrafenib has been FDA approved in patients with BRAF V600E/K-mutant metastatic melanoma, but cancer cell resistance typically occurred within a few months. Trametinib is an anti-cancer medication that acts as a MEK1 and MEK2 inhibitor, enzymes which are part of the same MAP kinase pathway. Trametinib has also been FDA approved in patients with BRAF V600E/K-mutant metastatic melanoma, but as with dabrafenib, cancer cell resistance typically occurred within a few months. To overcome this resistance, the above medications are often used together with good results. MAP kinase pathway inhibitors have been under investigation in the treatment of many different types of cancer, including cutaneous melanoma [1] Some drugs in this class have been associated with ocular side effects in up to $26.5 \%$ of patients, including retinal vein occlusion, multifocal serous retinal detachment, cystoid macular edema, and uveitis $[2,3]$. The mechanism behind these ocular side effects remains

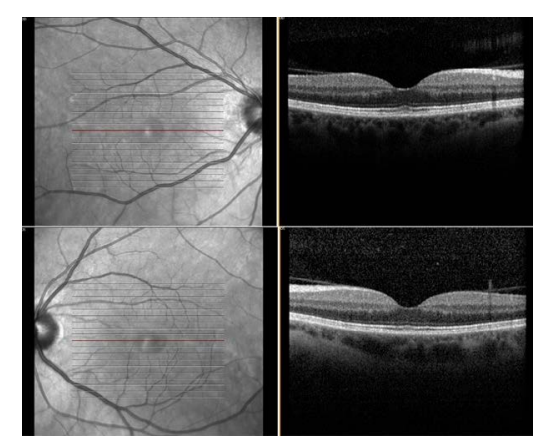

Figure 2. Resolution of previously noted retinal detachment.

Correspondence to: Kevin J. Talbot, Department of Ophthalmology, college of medicine, University of Florida, 1600 SW Archer Road, Gainesville, FL 32610, USA

Key words: dabrafenib; trametinib; B-Raf inhibitor; MEK1/2 inhibitor; stage IV melanoma; sub-foveal neurosensory detachment

Received: November 01, 2016; Accepted: November 08, 2016; Published: November 14, 2016 
unclear, but it has been suggested that MAP kinase inhibition can lead to an inflammatory response with consequent breakdown of the blood-retinal barrier [4]. This explanation is unlikely to account for the development of sub-foveal neurosensory detachment in our patient given the lack of inflammation on physical exam. To the best of our knowledge there has not been a case of isolated bilateral sub-foveal neurosensory retinal detachment due to simultaneous use of these two medication. With the advent of these new promising therapeutic anticancer modalities, some having a high rate of ocular side effects, regular ophthalmic evaluation and multi-disciplinary approach to customize individual patient treatment may be warranted [5-7].

\section{Declaration of interest}

The authors report no declarations of interest.

\section{References}

1. Leijen S, Middleton MR, Tresca P, Kraeber-Bodere F, Dieras V, et al. (2012) Phase I dose-escalation study of the safety, pharmacokinetics, and pharmacodynamics of the MEK inhibitor RO4987655 (CH4987655) in patients with advanced solid tumors. Clin Cancer Res 18: 4794-4805.

2. Duncan KE, Chang LY, Patronas M (2015) MEK inhibitors: a new class of chemotherapeutic agents with ocular toxicity. Eye (Lond) 29: 1003-1012.[Crossref]

3. Liu CY, Francis JH, Brodie SE, Marr B, Pulido JS, et al. (2014) Retinal toxicities of cancer therapy drugs: biologics, small molecule inhibitors, and chemotherapies. Retina 34: 1261-1280.[Crossref]

4. Huang W, Yang AH, Matsumoto D, Collette W, Marroquin L, et al. (2009) PD0325901, a mitogen-activated protein kinase kinase inhibitor, produces ocular toxicity in a rabbit animal model of retinal vein occlusion. J Ocul Pharmacol Ther 25:519-530. [Crossref]

5. Van der Noll R, Leijen S, Neuteboom GH, Beijnen JH, Schellens JH (2013) Effect of inhibition of the FGFR-MAPK signaling pathway on the development of ocular toxicities. Cancer Treat Rev 39: 664-672.

6. Joshi L, Karydis A, Gemenetzi M, Shao EH1, Taylor SR2 (2013) Uveitis as a Result of MAP Kinase Pathway Inhibition. Case Rep Ophthalmol 4: 279-282.[Crossref]

7. Renouf DJ, Velazquez-Martin JP, Simpson R, Siu LL, Bedard PL (2012) Ocular toxicity of targeted therapies. J Clin Oncol 30: 3277-3286.[Crossref]

Copyright: (2016 Talbot KJ. This is an open-access article distributed under the terms of the Creative Commons Attribution License, which permits unrestricted use, distribution, and reproduction in any medium, provided the original author and source are credited. 